\title{
Immunohistochemical Expression of Cytokeratins and Epithelial Membrane Protein 2 in Nasopharyngeal Carcinoma and its Potential Implications
}

\author{
Hussain Gadelkarim Ahmed ${ }^{1 *}$, Rania Saad Abdul Gader Suliman², Mohammed \\ Siddig Abd El Aziz ${ }^{2}$, Fawaz D Alshammari ${ }^{3}$
}

\begin{abstract}
Background: Nasopharyngeal carcinoma (NPC) is an aggressive disease and tends to involve surrounding tissues, and biomarkers for better management are yet to be identified. Materials and Methods: One hundred and fifty tissue samples with NPC diagnosis were were investigated using pan cytokeratin $(\mathrm{CK})$ and epithelial membrane protein 2 (EMP2) antibodies. Results: Immunohistochemical expression of CK was identified in 144/150 (96\%) and of EMP2 in 120/150 (80\%). Conclusions: There is a high loss of EMP2 in NPC, especially high grade examples. Loss of CK expression is also linked to high grade NPC types.
\end{abstract}

Keywords: Nasopharyngeal carcinoma - cytokeratin - EMP2 - immunohistochemistry

Asian Pac J Cancer Prev, 16 (2), 653-656

\section{Introduction}

In Sudan, there were 21,860 cases of all cancers diagnosed in 2008 , of which 2,942 cases were head and neck cancers; they represent $13.5 \%$ of all cancers (Ahmed et al., 2012; Mustafa et al., 2013). Nasopharyngeal carcinoma (NPC) is a cancer with unusually distinctive ethnic and geographic distributions, which is extremely predominant in Southern China and Southeast Asia (Jemal et al., 2011). Unlike other head and neck cancers, NPC often metastasizes to regional lymph nodes when diagnosed and has a tendency for the development of local recurrence after therapy (Razak et al., 2010). Three subtypes of NPC are recognized in the World Health Organization (WHO) classification: squamous cell carcinoma (I), typically found in the older adult population; non-keratinizing carcinoma (II); undifferentiated carcinoma (III) (Shanmugaratnam et al., 1991; Bernadette Brennan, 2006).

The exact molecular and morphological changes responsible for its high-aggressive potential behavior still mysterious. However, NPC is a malignant type of cancer due to its high occurrence rate of metastasis (Liu et al., 2014); however, biomarkers for effective diagnosis and treatment are yet to be identified.

Cytokeratins belong to the intermediate filament protein family. These biomarkers are useful in oncology diagnostics. At present, more than 20 different cytokeratins have been identified, of which cytokeratins 8, 18, and 19 are the most abundant in simple epithelial cells. Upon release from proliferating or apoptotic cells, cytokeratins provide useful markers for epithelial malignancies, distinctly reflecting ongoing cell activity (Barak et al., 2004. It has been suggested that cytokeratin 18 is a potential biomarker for the differentiation and prognosis of NPC, and its dysregulation might play an important role in the pathogenesis of NPC (Li et al., 2009). Therefore its expression is of interest.

EMP2 is a member of the four trans-membrane superfamily proteins and is believed to mediate transferring of miscellaneous proteins such as alpha6beta1 integrin and MHC class I to lipid raft micro domains. EMP2 has been recognized as a tumor suppressor gene in certain model systems. Normally, EMP2 is highly expressed at discrete locations in the body including high levels in the eye, lung, heart, thyroid, and uterus (Wadehra et al., 2003). Loss of EMP2 expression is common in NPC and it is associated with adverse prognosticators and might confer tumor aggressiveness (Yi-Hsien et al., 2012).

\section{Materials and Methods}

In this retrospective descriptive study 150 formalinfixed, paraffin embedded tissue blocks with their related data were retrieved from Radio Isotope Centre Khartoum. All of the 150 specimens were from patients previously diagnosed as having nasopharyngeal carcinomas.

${ }^{1}$ Department of Pathology, College of Medicine, ${ }^{3}$ Department of Medical Laboratory Science, College of Applied Medical Science, University of Hail, Hail, Kingdom of Saudi Arabia, ${ }^{2}$ Department of Histopathology and Cytology, Faculty of Medical Laboratory Science, Sudan University for Science and Technology, Sudan*For correspondence: hussaingad1972@yahoo.com 


\section{Sample processing}

Serial sections on poly-L-lysine-coated slides for immunohistochemistry (IHC) and one section on a regular slide for Hematoxylin and Eosin ( $\mathrm{H}$ and $\mathrm{E})$ staining procedure, were prepared from each tissue block. Sections were processed for immune-staining as follows:

The sections were de-paraffinized with xylene and then both sections were hydrated through $100 \%, 90 \%$, $70 \%$ and $50 \%$ ethanol sequentially. The sections, then, were treated for antigen retrieved by microwave treatment for 30 minutes in citrate buffer $(\mathrm{pH}$ 6.0). The slides were allowed cooling for 20 minutes in the citrate buffer before further treatment. After a quick rinse in phosphate buffered saline. Endogenous peroxidase was blocked by immersing slides in methanol with $0.3 \%$ hydrogen peroxide for 30 minutes (Dako k0411 kit).

The specimens were incubated in $5 \%$ goat serum for 10 minutes to block non-specific binding. Primary antibodies were incubated for 1 hour in a humidity chamber using the following dilutions: MIB-1 (DAKO Corp., Carpinteria, CA at a dilution of 1:50 ; COX-2 at a dilution of 1:50; polyclonal rabbit anti-(pan cytokeratin and EMP2) antibodies (Ab-1, Lab.Vision, Neo Markers, USA), Using antibody dilution at 10-20 $\mu \mathrm{g} \mathrm{mL-1}$ and incubated for 1 hour in a humid chamber, washed in phosphate-buffered saline (PBS) incubated for 30 minutes with the secondary biotinylated antibody followed by avidin peroxidase complex for another 30 minutes according to the manufacturer's instructions (Universal Detection Kit, Dako, Denmark). A brown color was developed with 3,3-diaminobenzidine tetra hydrochloride (DAB, Dako k0411 kit) for 5 minutes, washed in distilled water, and counterstained with Mayer's haematoxylin for 1 minute. The entire procedures were performed at room temperature. Additionally, a negative control for both markers, in which the primary antibody was omitted and replaced by phosphate buffered saline, was used. Positive control sections were added to process with the bladder tissue sections in the same run for precision and standardization of the elaborated IHC results.

\section{Ethical consent}

The study was approved by the Faculty Research Board of Sudan University for Science and Technology.

\section{Statistical analysis}

For all statistical analyses, the SPSS statistical software version 17 was used. Pearson chi square test was used and P. values of 0.05 or less were regarded as statistically significant.

\section{Results}

In this study, 150 tissue samples obtained from Sudanese patients with NPC were investigated. Patients' ages were ranging from 17 to 88 with a mean age of 50 years. Immunohistochemical expression of Cytokeratin was identified in 144/150 (96\%) and could not be identified in 6/150 (4\%). Epithelial Membrane Protein-2 was identified in $120 / 150(80 \%)$ and could not be identified in 30/150 (20\%). Of the 144 samples positive for CK, 120/144 (83.3\%) were also found to be positive for EMP2 and the remaining 24/144 (16.7\%) were negative. However, the $6 / 150$ (4\%) were found negative in CK, have also revealed loss of expression in EMP2, as indicated in Photomicrographs 1,2 and 3.

According to World Health Organization (WHO) classification of NPC, Type I, Type II, Type III, Type II and III, were identified in 0\%, 56/150(37.3\%), 85/150(56.7\%) and 9/150(6\%), respectively, as indicated in Figure 1.

Table 1 describes the distribution of the study population by NPC types and Immuno-expression of

Table 1. Distribution of the Study Population by NPC Types and Immunoexpression of Tumor Markers (CK and EMP2)

\begin{tabular}{lcccc}
\hline NPC type & \multicolumn{2}{c}{ CK } & \multicolumn{2}{c}{ EMP2 } \\
& Positive & Negative & Positive & Negative \\
\hline I & 0 & 0 & 0 & 0 \\
II & 56 & 0 & 48 & 8 \\
III & 81 & 4 & 67 & 18 \\
II\&III & 7 & 2 & 5 & 4 \\
Total & 144 & 6 & 120 & 30 \\
\hline
\end{tabular}

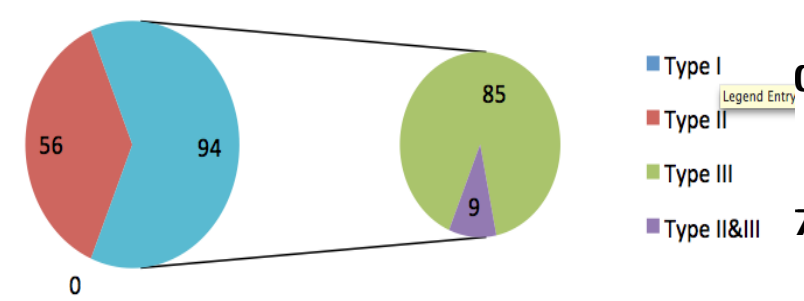

Figure 1. Description of the Study Population by NPC Types (WHO classification)

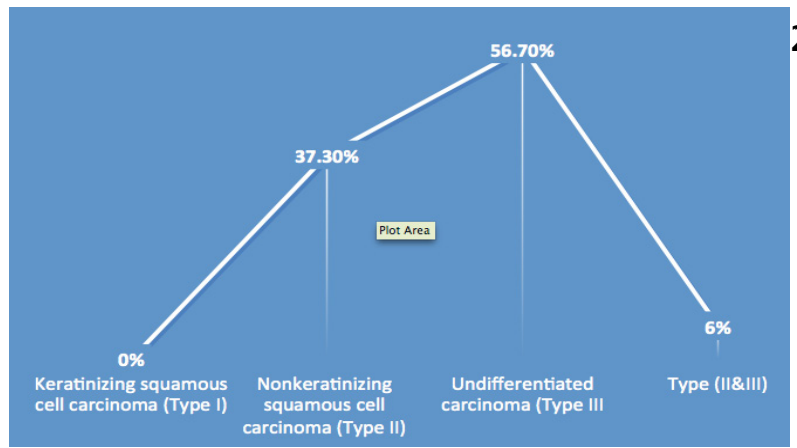

Figure 2. Description of the Study Population by NPC Histological Types

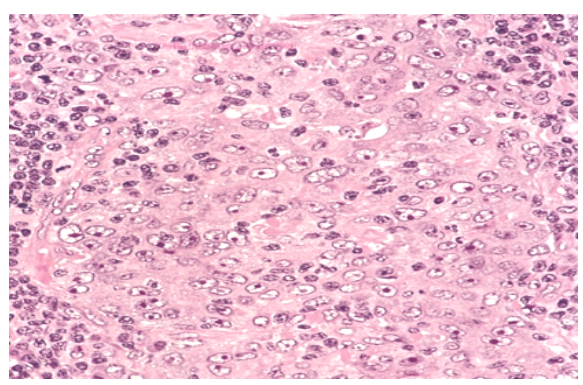

Figure 3. H\& E Stained Section of Nasopharyngeal Carcinoma, Obtained from 54 years Old Patient(x 40) 


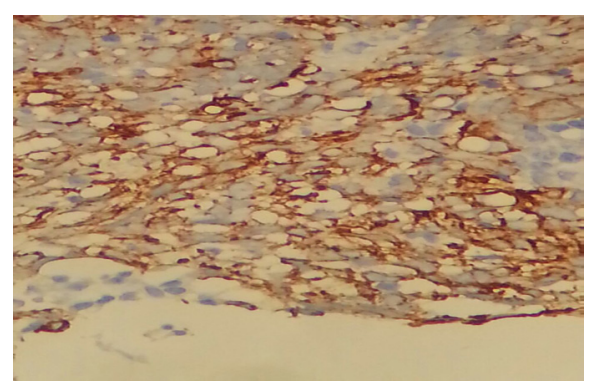

Figure 4. Formalin Fixed Paraffin Wax Embedded Section of Nasopharyngeal Carcinoma Immunostained for Cytokeratin Tumor Marker Using Water Bath Heating and Avidin Biotin Peroxidase Diaminobenzidine Labeling System, Show Cytoplasmic Positivity (x 40)

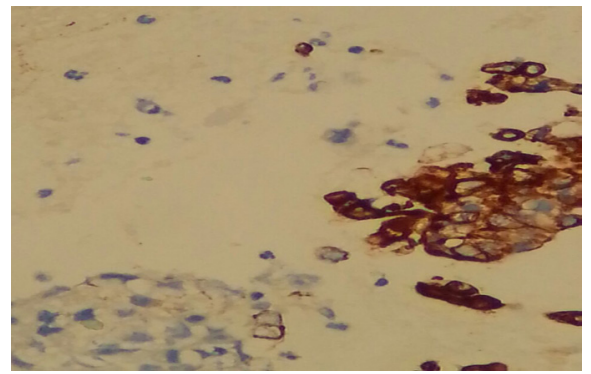

Figure 5. Formalin Fixed Paraffin Wax Embedded Section of Nasopharyngeal Carcinoma Immunostained for EMA Tumor Marker Using Water Bath Heating and Avidin Biotin Peroxidase Diaminobenzidine Labeling System, Show Cytoplasmic Positivity (x 40)

tumor markers (CK and EMP2). Negative CK expression was seen in advanced types. Of the six negative samples by CK, 4/6(66.7\%) were identified in Type III and the remaining 2/6(33.3\%) identified as showing both Types (II and III). Of the 30 specimens showing loss of expression of EMP2, 8/30 (26.7\%) were Type II, 18/30(60\%) were Type III and the remaining 4/30(13.3\%) were showing both Types (II and III), as indicated in Figure 1 and 2.

\section{Discussion}

Cancer is continuing to be a major challenging health problem in the Sudan (Ahmed 2012; Ahmed 2013). Although, there are numerous malignant tumors that may arise in the nasopharynx, NPC represent the most predominant type. NPC was subdivided according to WHO histopathological grading system into: Keratinizing squamous cell carcinoma (Type I), Nonkeratinizing squamous cell carcinoma (Type II) and Undifferentiated carcinoma (Type III, most common subtype) (Shanmugaratnam et al., 1991;Bernadette Brennan, 2006).

In the present study the most common subtype is Type III followed by Type II, which is similar to the findings of several studies (Richard et al., 2002).

The clinical importance of measuring soluble cytokeratin in body fluids lies in the early detection of recurrence and the fast assessment of the effectiveness of therapy response in epithelial cell carcinomas. The three most applied cytokeratin markers used in the clinic are tissue polypeptide antigen (TPA), tissue polypeptide specific antigen (TPS), and (cytokeratin 19 fragment) CYFRA 21-1. TPA is a broad spectrum test that measures Cytokeratins 8, 18, and 19. TPS and CYFRA 21-1 assays are more specific and measure cytokeratin 18 and cytokeratin 19, respectively. Although the main use of these markers is to monitor treatment and evaluate response to therapy, early prognostic information particularly on tumor progression and metastasis formation is also provided for several types of cancers. Cytokeratin tumor markers can accurately predict disease status before conventional methods and offer a simple, noninvasive, cheap, and reliable tool for more efficient management (Barak, 2004). Therefore, in the present study we found very high immunoexpression of CKs in NPC, which may indicate the release of some CK protein fragment in the blood. Consequently, such body fluids measures may be very important in early prediction of NPC cancerous change. This because NPC is difficult to detect early. That's probably because the nasopharnyx is not easy to examine and symptoms of nasopharyngeal carcinoma mimic those of other more common conditions (Keiji et al., 2011).

The positivity of tumor cells for pan-cytokeratin established the final diagnosis of non-keratinizing undifferentiated carcinoma (Boia et al., 2013). High levels of CK19-2G2 fragment expressed in tissue and serum are present in patients with NPC. The serum level of CK19$2 \mathrm{G} 2$ is supportive in the diagnosis of NPC. Furthermore, the combination of serum CK19-2G2 and EB-VCA IgA improves the detection sensitivity (Lei et al., 2012). Furthermore, it was suggested that cytokeratin 18 is a potential biomarker for the differentiation and prognosis of NPC, and its dis-regulation might play an important part in the pathogenesis of NPC.

EMP2 plays a tumor suppressor role through interacting with specific integrin(s) in epithelial cells and, thereafter, manages regular signaling transduction in benign conditions. Loss of EMP2 expression (49.2\%) was correlated with advanced primary tumor $(\mathrm{p}=0.044)$, nodal status $(\mathrm{p}=0.045)$ and the 7 th American Joint Committee on Cancer stage $(p=0.027)$. In multivariate analyses, loss of EMP2 expression emerged as an independent prognosticator for worse disease-specific survival (DSS; $\mathrm{p}=0.015$ ) and local recurrence-free survival (LRFS; $\mathrm{p}=0.030$ ), along with the American Joint Committee on Cancer stages III-IV ( $\mathrm{p}=0.034$, DSS; $\mathrm{p}=0.023$, LRFS) (Yi-Hsien et al., 2012). Although the exact features of the EMP2 protein in NPC progression continue to be clarified, the potential value of EMP2 as a prognostic biomarker in NPCs is guaranteed (Xu et al., 2011).

Nevertheless, and to the best of authors' knowledge, no previous study showed a correlation between CK and EMP2 immuno-expression in NPC or other cancer. However, from the major limitations in this study was the use of small number of CK negative samples, which didn't give us a reliable assessment of the exact relation between EMP2 and Pan CK.

Regarding the relationship between expression of CK and/or EMP2 and NPC subtypes, the loss of expression of both markers significantly seen in type III. Though 


\section{Hussain Gadelkarim Ahmed et al}

Subtype III represent the highest frequency in this study, there is still literature gap in this context.

In conclusion: There is a high loss of EMP2 in NPC, which is corresponding to high NPC types. Loss of CK expression is relatively linked to high NPC types. Further studies to highlight the biological interrelation between theses markers and NPC is deemed necessary.

\section{Acknowledgements}

The authors would like to thank people at histopathology at Radio Isotope Centre in Khartoum for helping in the sample collection. Also we would like to thank to Dr. Abolgasim Abass for helping in data analysis.

\section{References}

Abusail MS, Dirweesh AM, Salih RA, Gadelkarim AH (2013). Expression of EGFR and p53 in head and neck tumors among Sudanese patients. Asian Pac J Cancer Prev, 14, 6415-8.

Ahmed HG, Mustafa SA, Warille E (2012). Human papilloma virus attributable head and neck cancer in the Sudan assessed by p16INK4A immunostaining. Asian Pac J Cancer Prev, 13, 6083-6.

Ahmed HG (2012). Awareness survey on knowledge of microbial infectious causes of cancer in Northern State of Sudan. Asian Pac J Cancer Prev, 3, 5497-500.

Ahmed HG (2013). Survey on knowledge and attitudes related to the relation between tobacco, alcohol abuse and cancer in the Northern State of Sudan. Asian Pac J Cancer Prev, 14, 3345-8.

Barak V, Goike H, Panaretakis KW, Einarsson R (2004). Clinical utility of cytokeratins as tumor markers. Clin Biochem, 37, 529-40.

Bernadette Brennan (2006). Nasopharyngeal carcinoma. Orphanet J Rare Dis, 1, 23.

Boia ER, Boia M, Balica NC, et al (2013). Non-keratinizing undifferentiated carcinoma of the nasopharynx. Rom $J$ Morphol Embryol, 54, 839-43.

Jemal A, Bray F, Center MM, et al (2011). Global cancer statistics. CA Cancer J Clin, 61, 69-90.

Tabuchi K, Nakayama M, Nishimura B, Hayashi K, Hara A. (2011). Early detection of nasopharyngeal carcinoma. Int $J$ Otolaryngol, 2011, 638058

Lei DS, Yu J, Tong XL, Wang MW, Wang K, Chen H (2012). Diagnostic value of cytokeratin 19 fragment in nasopharyngeal carcinoma. Zhonghua Bing Li Xue Za Zhi, 41, 461-5.

Li XM, Huang WG, Yi H, Cheng AL, Xiao ZQ (2009). Proteomic analysis to identify cytokeratin 18 as a novel biomarker of nasopharyngeal carcinoma. J Cancer Res Clin Oncol, 135, 1763-75.

Liu A, Huang W, Zeng G, et al (2014). Expression of the Annexin A1 gene is associated with suppression of growth, invasion and metastasis of nasopharyngeal carcinoma. Mol Med Rep, 10, 3059-67.

Razak AR, Siu LL, Liu FF, Ito E, O'Sullivan B et al (2010). Nasopharyngeal carcinoma: the next challenges. Eur $J$ Cancer, 46, 1967-8.

Richard Cote, Saul Suster, Lawrence Weiss, Noel Weidner (Editor) (2002). Modern Surgical Pathology (2 Volume Set). London: W B Saunders. ISBN 0-7216-7253-1.

Shanmugaratnam K, Sobin L H, Barnes L et al. (1991). World Health Organization Histological Classification of Tumours. Histological typing of tumours of the upper respiratory tract and Ear, 2nd edn. Springer-Verlag, Berlin Heidelberg, 32-3. Wadehra M, Sulur GG, Braun J, Gordon LK, Goodglick L (2003). Epithelial membrane protein-2 is expressed in discrete anatomical regions of the eye. Exp Mol Pathol, 74, 106-12.

Xu HM, Liang Y, Chen Q, et al (2011). Correlation of Skp2 overexpression to prognosis of patients with nasopharyngeal carcinoma from South China. Chin J Cancer, 30, 204-12.

Yi-Hsien Chen, Li-Ching Wu, Wen-Ren Wu, et al (2012). Loss of epithelial membrane protein-2 expression confers an independent prognosticator in nasopharyngeal carcinoma: a cohort study. BMJ Open, 2, 900. 